\title{
Atuação do profissional de Educação Física na equipe multidisciplinar do \\ Consultório na Rua em Aracaju/SE: Relato de experiência
}

Physical Education professional's performance in the multidisciplinary team of the Consultancy on the Street in Aracaju/SE: Experience report

Desempeño del profesional de Educación Física en el equipo multidisciplinario de la Consultoría en la Calle de Aracaju/SE: Informe de experiencia

Jadisson Gois da Silva

ORCID: https://orcid.org/0000-0003-0089-4852 Universidade Federal de Sergipe, Brasil

E-mail: jadissoned.fisica2014@outlook.com

Rosa Luciana Prado

ORCID: https://orcid.org/0000-0002-3842-3493 Centro Universitário Estácio de Sergipe, Brasil E-mail: rosa_luciana@hotmail.com

Flávia Menezes Almeida

ORCID: https://orcid.org/0000-0001-7690-0409 Universidade Federal de Sergipe, Brasil E-mail: flaviiamenezes@hotmail.com

Maria de Lourdes Barros Avelino ORCID: https://orcid.org/0000-0001-5170-1227 Universidade Federal de Sergipe, Brasil E-mail: avelinomld@gmail.com

Jayane Pinheiro Trindade

ORCID: https://orcid.org/0000-0002-1024-1647 Universidade Federal de Sergipe, Brasil E-mail: jayanetrindade9@gmail.com

Tharciano Luiz Teixeira Braga da Silva ORCID: https://orcid.org/0000-0001-5467-7875 Centro Universitário Estácio de Sergipe, Brasil E-mail: tharcianoluiz@gmail.com

Cristiano Mezzaroba

ORCID: https://orcid.org/0000-0003-4214-0629 Universidade Federal de Sergipe, Brasil

E-mail: cristiano_mezzaroba@yahoo.com.br.com

\section{Resumo}

O presente estudo tem como objetivo apresentar as experiências vividas por um Profissional de Educação Física (PEF) no exercício de suas funções numa equipe de Consultório na Rua (CR). Trata-se de um relato de experiência, acerca das vivências de um PEF residente Multiprofissional em Saúde Mental ao atuar no CR em Aracaju/SE, entre agosto de 2019 a fevereiro de 2020. Para a coleta dos dados utilizou-se a observação participante com os dados sendo elaborados em um diário de campo. Identificamos situações de preconceito e discriminação por parte da sociedade acerca da população em situação de rua; dificuldades de acesso à Rede de Atenção à Saúde (RAS); extrema vulnerabilidade social e notórias lacunas entre as políticas públicas de saúde e a sua aplicabilidade. Verificou-se a importância do trabalho da equipe do CR - um trabalho de cuidado e assistência desenvolvendo ações integradas com os serviços da RAS. Além disso, destaca-se a relevância da inserção do PEF no CR como agente que atua na perspectiva da integralidade do cuidado, desenvolvendo estratégias de promoção à saúde para além do seu núcleo de especificidade. Ademais, este atua frente às diferentes problemáticas e necessidades de saúde da referida população, inclusive na busca ativa aos usuários que fazem uso de drogas, contribuindo, também, para a realização do apoio matricial, acolhimento, educação em saúde, articulação intersetorial, e estratégias de redução de danos. Portanto, a Residência, torna-se uma potente estratégia para proporcionar processos de ensino e aprendizagens com vistas a uma formação continuada.

Palavras-chave: Atenção primária à saúde; Educação superior; Consultório na rua; Educação física. 


\begin{abstract}
This study aims to present the experiences lived by a Physical Education Professional (PEF) in the exercise of their functions in a team of Consultório na Rua (CR). It is an experience report, about the experiences of a Multiprofessional resident PEF in Mental Health while working at the RC in Aracaju/Sergipe/Brazil, between August 2019 and February 2020. For data collection, participant observation with the data being prepared in a field diary. We identified situations of prejudice and discrimination on the part of society regarding the homeless population; difficulties in accessing the Health Care Network (RAS); extreme social vulnerability and notorious gaps between public health policies and their applicability. The importance of the work of the CoR team was verified - a work of care and assistance developing actions integrated with the services of the SAN. In addition, the relevance of the insertion of the PEF in the CR is highlighted as an agent that acts from the perspective of comprehensive care, developing health promotion strategies beyond its specificity core. Furthermore, it acts in the face of the different health problems and needs of that population, including the active search for users who drugs, also contributing to the realization of matrix support, reception, health education, intersectoral articulation and strategies harm reduction. Therefore, the Residence, becomes a powerful strategy to provide teaching and learning processes with a view to continuing education.
\end{abstract}

Keywords: Primary health care; Higher education; Street office; Physical education.

\title{
Resumen
}

Este estudio tiene como objetivo presentar las experiencias vividas por un Profesional de Educación Física (PEF) en el ejercicio de sus funciones en un equipo de Consultório en la Calle (CC). Este es un relato de experiencia sobre las vivencias de un PEF multiprofesional residente en Salud Mental mientras trabajaba en el CC en la ciudad de Aracaju/SERGIPE/BRASIL, entre agosto de 2019 y febrero de 2020. Para la recolección de datos, se utilizó la observación participante y los datos se prepararon en un diario de campo. Identificamos situaciones de preconcepción y discriminación por parte de la sociedad hacia la población sin hogar; dificultades para acceder a la Red de Atención Sanitaria (RAS); extrema vulnerabilidad social y notorias brechas entre las políticas públicas de salud y su aplicabilidad. Se verificó la importancia del trabajo del equipo de CC - un trabajo de atención y asistencia desarrollando acciones integradas con los servicios de la SAN. Además, se destaca la relevancia de la inserción del PEF en la CC como un agente que actúa desde la perspectiva de la atención integral, desarrollando estrategias de promoción de la salud más allá de su núcleo de especificidad. Además, actúa frente a los diferentes problemas y necesidades de salud de esa población, incluyendo la búsqueda activa de usuarios que de drogas, contribuyendo también a la realización de matrices de apoyo, recepción, educación en salud, articulación intersectorial y estrategias de reducción de daños. Por tanto, la Residencia, se convierte en una poderosa estrategia para dotar de procesos de enseñanza y aprendizaje con miras a la formación continua.

Palabras clave: Atención primaria de salud; Educación superior; Consultoría en la calle; Educación física.

\section{Introdução}

As Residências Multiprofissionais em Saúde foram introduzidas e regulamentadas pela promulgação da lei nº 11.129 , de 30 de junho de 2005. Trata-se de uma modalidade de pós-graduação lato sensu equivalente a uma carga horária de 5.760 horas, sendo $20 \%$ teórica e $80 \%$ prática, voltada para a educação em serviço, destinada às categorias profissionais que integram a área de saúde, excetuada a categoria médica (Brasil, 2006).

Nesse sentido, a Residência Multiprofissional em Saúde Mental do Hospital Universitário da Universidade Federal de Sergipe (HU-UFS) desenvolve-se nessa mesma perspectiva e a formação em serviço ocorre nas redes conveniadas de saúde, mais precisamente, na Rede de Atenção Psicossocial do município de Aracaju, Sergipe, sob supervisão de preceptores. A equipe multiprofissional do programa de Saúde Mental é composta por cinco categorias de profissões, a saber: Enfermagem, Serviço Social, Farmácia, Psicologia e Educação Física, em seu processo seletivo são aprovados dois profissionais de cada área, formando assim duas equipes de Residentes.

Durante o processo de experiência na Residência Multiprofissional em Saúde Mental foram vivenciados os seguintes serviços de saúde pública ${ }^{1}$ : Centro de Atenção Psicossocial (CAPS) III Transtorno, Centro de Atenção Psicossocial Infanto-

\footnotetext{
${ }^{1}$ Conforme o Artigo 198 da Constituição de 1988, as ações e serviços públicos de saúde integram uma rede regionalizada e hierarquizada e constituem um sistema único, organizado de acordo com as seguintes diretrizes: I - descentralização, com direção única em cada esfera de governo; II - atendimento integral, com prioridade para as atividades preventivas, sem prejuízo dos serviços assistenciais; III - participação da comunidade.
} 
juvenil (CAPSi) Transtorno, Centro de Atenção Psicossocial Infanto-juvenil (CAPSi) Álcool, Crack e outras Drogas, Projeto de Redução de Danos (PRD) e Consultório na Rua. Neste relato de experiência, temos o Consultório na Rua como foco deste estudo, haja vista que dentre os equipamentos de saúde o Consultório na Rua despertou-me notório interesse pelo o assunto, levando-se em consideração a sua peculiaridade.

O Consultório na Rua é formado por equipes multiprofissionais e prestam atenção integral à saúde de uma referida população em situação de rua, em vista disso, a criação e implementação do serviço Consultório na Rua no Município de Aracaju, Sergipe, vem trazer um trabalho de cuidado e assistência à população em situação de rua de forma estruturada, desenvolvendo ações compartilhadas e integradas com a Unidade Básica de Saúde e com as equipes dos CAPS, dos serviços de Urgência e Emergência e de outros pontos de atenção à saúde (Vasconcelos, 2015). Desta forma, a equipe do Consultório na Rua busca atuar frente às diferentes problemáticas e necessidades de saúde da população em situação de rua, inclusive na busca ativa e cuidado aos usuários que fazem uso "problemático" de álcool e outras drogas (Brasil, 2009).

O Sistema Único de Saúde (SUS)² caracteriza-se como uma porta de entrada para o Profissional de Educação Física fortalecer a sua prática e contribuir no processo de cuidado em saúde, e nesse sentido, cenários diversos de atuação oportunizam novas construções e a ressignificação de saberes historicamente construídos, a exemplo da influência militar e biomédica, bem como da higienização e da eugenização (Varela \& Oliveira, 2018).

Nesse sentido, observa-se que as residências multiprofissionais cumprem um importante papel nesse processo, pois se propõem a atender as necessidades socioepidemiológicas da população, potencializando a formação a partir da inserção de equipes multiprofissionais nos diversos serviços de saúde, portanto, a residência tem se mostrado uma potente estratégia para apresentar o papel da Educação Física no campo da saúde mental (Brasil, 2006; Varela \& Oliveira, 2018).

Diante as informações supracitadas, este relato de experiência tem como tônica estabelecer uma reflexão crítica acerca do processo de formação em Educação Física, buscando contribuir com as discussões e ampliar a gama de informações acerca das possibilidades de atuação e subsequente intervenção no campo da saúde coletiva ${ }^{3}$. Sendo assim, ao que concerne o seu objetivo, este tem como intuito, apresentar as experiências vividas por um Profissional de Educação Física no exercício de suas funções numa equipe de Consultório na Rua no município de Aracaju/SE, enquanto Residente Multiprofissional em Saúde Mental da Universidade Federal de Sergipe.

\section{Metodologia}

Trata-se de um estudo descritivo de abordagem qualitativa do tipo relato de experiência.

De acordo com Godoy (1995) “[...] a pesquisa qualitativa ocupa um reconhecido lugar entre as várias possibilidades de se estudar os fenômenos que envolvem os seres humanos e suas intrincadas relações sociais, estabelecidas em diversos ambientes (p. 21) [...]".

Segundo Oliveira, Marques e Schreck (2017) o relato de experiência caracteriza-se como a descrição que um autor ou até mesmo uma equipe profissional utiliza-se com intuito de relatar suas vivências, percebendo-as como positiva ou negativa, contribuindo para discussões e trocas de sugestões de assunto que sirvam para aperfeiçoamento dos cuidados ao que tange a saúde dos sujeitos envolvidos com/na experiência da ação realizada.

\footnotetext{
2 É considerado a maior conquista social do povo brasileiro, sendo também uma ação reacionária à ditadura militar e, por último e não menos importante, esse sistema é fruto da reforma sanitária brasileira, sendo, portanto, caracterizado como um conjunto de ações e serviços de saúde, prestados por órgãos e instituições públicas, estaduais e municipais, da administração direta e indireta e das fundações mantidas pelo Poder Público (Barros, 2015).

${ }^{3}$ Campo de produção de conhecimento especializado, interdisciplinar, com subáreas da saúde pública, da epidemiologia e da medicina preventiva e social que vem com a finalidade de fomentar discussões e reencontros de relevância social com caráter técnico científico de profissionais da saúde (Carvalho \& Ceccim, 2006).
} 
Nesse sentido, o cenário onde se desenvolveu o estudo foi o Consultório na Rua ${ }^{4}$, que tem como sede administrativa a Unidade Básica de Saúde Maria do Céu, localizado no município de Aracaju, Sergipe, mais precisamente, na região central da cidade, onde a população em situação de rua tem histórico de acesso. É oportuno frisar que o Consultório na Rua é cenário de prática da Residência Multiprofissional em Saúde Mental do HU-UFS.

O agente do referido estudo foi um Profissional de Educação Física Residente Multiprofissional em Saúde Mental que permaneceu na equipe do mencionado serviço de saúde no período compreendido entre o mês de agosto de 2019 a fevereiro de 2020, contendo uma carga horária de 3 turnos semanais: quarta-feira turno vespertino, quinta-feira turno vespertino e noturno. $\mathrm{Na}$ experiência ora relatada, a equipe fora composta por uma Residente em Saúde Mental com formação em psicologia, e, uma assistente social, uma enfermeira, duas técnicas de enfermagem e um médico (ambos da equipe do Consultório na Rua vinculados à Prefeitura Municipal de Aracaju).

Durante a referida experiência alguns profissionais foram saindo do Consultório na Rua devido a questões burocráticas de contrato, a exemplo de uma técnica de enfermagem e do médico da equipe, sendo que, já próximo à finalização da experiência no referido cenário, foi contratada por vias de processo seletivo vigente uma psicóloga para compor a equipe; sendo importante pontuar que essa foi a primeira vez que a equipe do Consultório na Rua recebera um Residente com formação em Educação Física ${ }^{5}$.

Para a efetivação da coleta dos dados utilizou-se como estratégia a observação participante que permitiu compreender e descrever o objeto da pesquisa e para a sistematização e análise dos dados obtidos nas experiências vividas foi elaborado um diário de campo contendo o registro das atividades (Minayo, 2004). Por fim, foram descritas as atividades realizadas de forma sintética em que se objetivou articular a teoria e a prática, a reflexão crítica e o processo de autoaprendizagem.

Outra ferramenta metodológica utilizada para melhor descrever a experiência foram os registros fotográficos realizados durante o período de vivência dos processos de trabalho. Para Minayo (2004), enquanto registros complementares, as fotografias permitem documentar momentos ou situações que ilustram o cotidiano vivenciado, o que oportuniza a ampliação do conhecimento do estudo e mostra o contexto do universo pesquisado para além do que as palavras escritas podem alcançar.

\section{Resultados e Discussão}

Durante o tempo de percurso na equipe do Consultório na Rua identificamos e experiênciamos situações de preconceitos e discriminação por parte da sociedade acerca da população em situação de rua. Sendo perceptível que isso ocorre devido à aparência das pessoas em situação de rua que, por vezes, vestem-se com roupas rasgadas e sujas, até mesmo pela dificuldade de acesso a insumos para sua própria higiene, levando-se em consideração suas peculiaridades, sendo passíveis de diversos estigmas ${ }^{6}$ sociais.

Tais preconceitos acabam por vezes excluindo aqueles considerados "diferentes", o que se torna preocupante, uma vez que, em um país como o Brasil, onde a miscigenação é a tônica da sociedade, a mesma utiliza-se de ações indesejáveis e assim exclui os que fazem parte da diversidade, o que evidencia a brutal desigualdade social de uma das maiores economias mundial.

\footnotetext{
${ }^{4}$ A estratégia Consultório na Rua foi instituída pela Política Nacional de Atenção Básica, em 2011, e visa ampliar o acesso da população em situação de rua aos serviços de saúde, ofertando, de maneira mais oportuna, atenção integral à saúde para esse grupo populacional, o qual se encontra em condições de vulnerabilidade e com os vínculos familiares interrompidos ou fragilizados. O CR do Município de Aracaju/SE iniciou seu processo de trabalho em 2 de junho de 2015. Sendo composto por equipe multidisciplinar, cumprindo carga horária mínima semanal de 30 horas, tendo horário de funcionamento adequado às demandas das pessoas em situação de rua (Vasconcelos, 2015).

5 Somente no ano de 2019 é que o CR tornou-se cenário de prática da Residência em Saúde Mental do HU-UFS, relevante pontuar que a partir do ano de 2019, somente houve atuação de um Profissional de Educação Física neste serviço. Além desses fatos, o CR já recebera graduandos de outros núcleos de formação, a saber: Enfermagem, Serviço Social e Psicologia.

${ }^{6}$ Segundo Melo (2000), estigmas, para Erving Goffman, são identidades deterioradas, por uma ação social, que representam algo "mal" dentro da sociedade e, por isso, deve ser evitado.
} 
Vasconcelos (2015) destaca que as pessoas em situação de rua apresentam determinadas características que devem ser demasiadamente enfrentadas, a exemplo disso: a questão da violência e vulnerabilidade, alimentação incerta e em baixas condições de higiene, água de baixa qualidade e pouco disponível, privação do sono, privação de afeição, variações climáticas, falta de tempo para buscar atendimento para o cuidado e o autocuidado da saúde, vergonha, internação e alta em serviços de saúde e adesão ao tratamento com subsequente acompanhamento.

Para tanto, é necessário, antes de tudo, rejeitar os estereótipos que aprisionam e fixam os indivíduos, os quais os classificam como feio ou bonito, capaz ou incapacitado, saudável ou doente, normal ou anormal (Goellner, 2010). Ainda conforme Goellner (2010) "Diferença não significa desigualdade e essa só pode ser minimizada se houver iniciativas que promovam atividades co-oparticipativas, nas quais as diferenças não sejam eliminadas, mas tratadas em suas especificidades" (p.79). Além disso, também foi perceptível a notória lacuna entre as políticas públicas de saúde e a sua aplicabilidade.

A exemplo do exposto destaca-se a Política Nacional de Atenção Básica ${ }^{7}$, bem como a Política Nacional de Humanização ${ }^{8}$, tendo em vista que, por vezes, o usuário em situação de rua encontra barreiras para ter o devido acesso à Unidade Básica de Saúde, seja pelo fato de não estar com documento de identidade, seja por estereótipos, ou até mesmo em razão de não ter um acolhimento que corresponda aos princípios expressamente apresentados na Constituição Federal de 1988, como também na Lei Orgânica da Saúde nº 8.080/90 (consolidação da universalidade do acesso, a equidade, ofertando mais a quem tem menos e a integralidade do cuidado, ou seja, garantia do direito ao cidadão a ser atendido conforme a sua necessidade) (Brasil, 1988; Brasil, 1990; Machado et al., 2007).

No entanto, observa-se que as condições de vulnerabilidade vivenciadas pelas pessoas em situação de rua, como também as questões psicossociais geradoras de intensos sofrimentos psíquicos, promovem riscos maiores para a saúde desse grupo, representando um desafio à efetivação de políticas de saúde que deem conta dessa complexidade, o que demonstra que a referida população necessita de um maior suporte por parte dos diversos setores da saúde e dentre outros, buscando assim a efetivação do princípio da equidade, o qual considera tratar diferentemente os desiguais, ou seja, promover a justiça social (Brasil, 1988; Vasconcelos, 2015).

Nesse sentido, torna-se oportuno frisar que o acolhimento representa uma postura ética, implicando no compartilhamento de saberes, necessidades, possibilidades, angústias e invenções, pois compete ao profissional escutar as queixas, medos e expectativas dos usuários e com isso identificar riscos e vulnerabilidades, e assim tecer encaminhamentos de maneira responsável e resolutiva (Brasil, 2009). Segundo Zappelini e Oliveira (2007) a ação de acolher deve começar desde a recepção do serviço e perpassar todo o processo de trabalho, envolvendo tanto os trabalhadores quanto os usuários com vistas à construção de vínculos bem como a responsabilização do cuidado em saúde.

Assim, destacamos que o acolhimento ocorria dentro da sala do Consultório na Rua e/ou na UBS Maria do Céu, sendo, portanto, realizado por profissionais que compõem a equipe do Consultório na Rua e/ou Residentes em Saúde Mental, neste sentido, visava-se acolher na perspectiva da imparcialidade, levando-se em consideração o histórico e trajetória de vida das pessoas.

Segundo Ayres (2006) poder ouvir e fazer-se ouvir são a essência de um diálogo com o objetivo de alcançar o cuidado em saúde, a ênfase está na qualidade da escuta que busca ir além das demandas explícitas, que correlaciona o contexto do usuário e fornece a este condições de refletir sobre seu próprio modo de vida, contribuindo para um cuidado integral em saúde.

\footnotetext{
7 Define a organização em Redes de Atenção à Saúde (RAS) como estratégia para um cuidado integral e direcionado às necessidades de saúde da população, destaca a Atenção Básica/Primária como primeiro ponto de atenção e porta de entrada preferencial do Sistema Único de Saúde, que deve ordenar os fluxos e contrafluxos de pessoas, produtos e informações em todos os pontos de atenção à saúde (Brasil, 2008).

8 Também conhecida como HumanizaSUS, foi criada em 2003 e preconiza sobre a conduta do profissional de saúde, nas relações interpessoais e sobre o ambiente de saúde que deve proporcionar um acolhimento ao usuário. (Barros, 2015).
} 
Desta forma, pensar em integralidade é também vislumbrar o processo formativo de profissionais para o que concerne à saúde coletiva, preparando-os para o trabalho em equipe, fazendo uso dos saberes nucleares de cada categoria profissional de forma horizontal, tendo o olhar voltado para os determinantes sociais em saúde e não somente para a doença e o seu tratamento (Akerman \& Feurwerker, 2006). Visto que, saúde em uma concepção integral e ampliada, representa a possibilidade de visualizar o indivíduo na sua singularidade, ou seja, levando-se em consideração os determinantes sociais que influenciam significativamente na qualidade de vida (Waal et al., 2009).

Assim, durante o processo de vivência no Consultório na Rua, utilizava-se o conceito de saúde para além de uma visão biológica, portanto, considerava-se a vivência do indivíduo em sociedade, bem como o acesso às redes socioeconômicas e de serviços essenciais, uma vez que o modelo de atenção pautado no cuidado integral, compartilhado, e na comunidade é de suma importância acerca de uma assistência de qualidade.

Ressalta-se que os encaminhamentos impressos realizados pela equipe do Consultório na Rua aos outros serviços eram avaliados de forma satisfatória pelo usuário, pois facilitavam o acesso destes. Vale-se mencionar que os usuários contavam com o importante processo de trabalho da equipe do Consultório na Rua, tendo em vista um trabalho pautado na resolubilidade das problemáticas encontradas, justamente, para transpor as barreiras de acesso a outros equipamentos de saúde. Portanto, com o apoio da assistente social providenciava-se os documentos dos usuários que, neste processo, era realizado os devidos encaminhamentos para a segunda via da Certidão de Nascimento, confecção de RG, CPF, Carteira de Trabalho e até mesmo quando o usuário necessitava do Cartão do SUS era realizada a devida confecção.

Aqui cabe destacar, que a possibilidade de um trabalho multidisciplinar traz o Profissional de Educação Física para atuar nesse espaço, cabendo-o, em sua prática, ser um dos agentes para o processo de promoção à saúde ${ }^{9}$, visualizando o sujeito em suas múltiplas dimensões. Tornando-se perceptível a relevância da minha inserção enquanto profissional de Educação Física no Consultório na Rua como sujeito que atua na perspectiva da integralidade do cuidado.

Neste sentido, para além do núcleo de especificidade, a exemplo da oferta da atividade física/práticas corporais, tornou-se possível atuar frente às diferentes problemáticas e necessidades de saúde da referida população em conjunto com a equipe do Consultório na Rua, inclusive na busca ativa e cuidado aos usuários que fazem uso "problemático" de álcool e outras drogas.

Sendo assim, durante a vivência no mencionado serviço de saúde fui um dos agentes que contribuiu para a realização do apoio matricial. Oliveira e Wachs (2018) referem que o apoio matricial pode ser entendido como uma tecnologia em saúde que envolve um rearranjo organizacional da gestão do trabalho, por meio da relação entre equipe de referência e apoiador matricial. Além disso, Cruz e Ferreira (2007) apontam que esta tecnologia representa o encontro de profissionais para discussão de casos e realizar a troca de saberes referente à determinada temática ou até mesmo um usuário e suas mais diversas necessidades.

Cabe elucidar que durante a inserção no referido serviço de saúde observei que o apoio matricial acontecia intrasetorialmente - entre equipes de saúde, e intersetorialmente - profissionais de saúde com outros profissionais, como por exemplo, da assistência social, da educação etc. Logicamente que a depender de cada caso e suas necessidades.

A exemplo disso, e dada a relevância, destaco a situação de um usuário (para resguardar o anonimato não será mencionado o nome deste) que em situação de grande vulnerabilidade social, estando este dormindo na calçada de uma borracharia e fazendo uso abusivo de álcool e outras drogas (crack e cannabis - maconha) sem acesso à alimentação e a

\footnotetext{
9 “A promoção à saúde está determinada pelo desenvolvimento econômico, pela distribuição equitativa da renda, pelo lazer, pela educação sanitária, pela alimentação, nutrição e pelo exercício físico" (Carvalho, 2001, p.18).
} 
insumos para sua própria higiene foram realizados manejo na Urgência Mental e matriciamento com o CAPS AD Primavera, tendo ênfase na longitudinalidade ${ }^{10}$ do cuidado.

Nesse ínterim, a intersetorialidade é entendida como estratégia em que os diferentes setores sociais, com seus saberes e práticas precisam se articular e se integrar com vistas a orientar e garantir a integralidade do cuidado (Junqueira, 2000). Realizamos tais articulações com o Centro de Atenção Psicossocial Álcool e outras Drogas (CAPSad) Primavera; Centro de Atenção Psicossocial Infanto-juvenil (CAPSi) Álcool, Crack e outras Drogas; Unidade de Acolhimento para Adultos (UAA), Casas de Passagem; Unidades Básicas de Saúde, Centro POP e Projeto de Redução de Danos (PRD). Nestas articulações estive como sujeito em processo de aprendizagem, participando das discussões dos casos, e em determinados momentos compartilhando das vivências adquiridas com os usuários e suas diversas necessidades.

Portanto, eram compartilhados os sentimentos, emoções e percepções acerca da trajetória e histórico de vida dos usuários que, por vezes, traziam a partir dos excertos de suas falas e pelas características faciais, diversos sofrimentos psíquicos, tendo como tônica a questão dos laços familiares fragilizados ou até mesmo interrompidos, além disso, havia fortemente marcado a questão do uso de álcool e outras drogas.

Releva mencionar que durante as abordagens aos usuários trabalhava-se com educação em saúde, tanto referente aos cuidados a serem tomados frente ao uso de drogas, como também acerca do uso do preservativo nas relações sexuais; tal educação em saúde também era realizada quando a equipe do Consultório na Rua realizava testes rápidos: Sífilis, HIV, Hepatites A e B. Portanto, além da educação em saúde (a partir de uma perspectiva integrada considerando as questões sociais e não apenas biológicas/biomédicas), verifica-se, a partir deste, a redução de danos.

Silveira (2012) destaca a educação em saúde como estratégia de promoção à saúde neste processo de conscientização individual e coletiva de responsabilidades e de direitos à saúde, estimulando ações que atendam aos princípios norteadores do SUS. A partir deste enfoque, observa-se a importância de eleger estratégias didáticas que conduzam a uma transformação dos usuários ampliando sua capacidade de compreensão acerca de uma vida mais saudável a partir de contextos mais ampliados de saúde.

E ao que concerne a redução de danos, Vinadé, Cruz e Barbeito (2014) assinalam que as estratégias de redução de danos têm o intuito de redimensionar o olhar sobre a droga versus o usuário de substâncias psicoativas com o objetivo de romper o preconceito da sociedade, ao colocar o usuário apenas como "drogado", mas dirige-se para um campo de ruptura com a centralidade da droga sobre a vida das pessoas.

Já referente aos atendimentos internos (neste caso na própria sede do Consultório na Rua) realizava-se acolhimento ao público que procurava a unidade por demanda espontânea, nesse sentido, era realizado escuta ativa ${ }^{11}$; identificação da demanda e a necessidade do usuário; orientações e encaminhamentos, com enfoque na inclusão destes usuários na Rede de Atenção à Saúde. Ademais, participava-se das atividades internas, tais como: reuniões de equipes, discussões de caso, planejamento das atividades semanais, e não menos importante compartilhamento dos casos de matriciamento.

Frente às atividades externas destacam-se as múltiplas tentativas de aproximação com os usuários, nesse sentido, havia a disponibilidade e o desejo acerca do cuidado em saúde mental, utilizando-se da arte como recurso terapêutico, a exemplo do exposto, releva pontuar, que era feito uso de cartazes, tintas, e violão, nesse ínterim, havia o estímulo para

\footnotetext{
${ }^{10}$ Consiste no acompanhamento do usuário ao longo do tempo, pressupõe-se a existência de uma fonte continuada de atenção, com a presença de vínculo, relação interpessoal e de confiança entre usuários e profissionais de saúde (Kessler et al., 2019).

11 É uma modalidade de observação atenta de cada elemento incluído na relação (a si mesmo, com o outro, com o contexto, com o particular e com a forma), incluindo de forma explícita o estado de reflexão como parte do processo (Sclavi, 2003 citado por Moura \& Giannella, 2016). “O propósito não é apenas ouvir para registrar uma opinião, é compreender o que está por trás, para além da aparência, uma porta para o diálogo e revisão das próprias certezas, uma chance de enxergar outras possibilidades e modos de ser das coisas" (Moura \& Giannella, 2016, p.2).
} 
construir com os usuários: músicas, canções, poesias e pinturas sobre suas histórias de vida, com enfoque no respeito à autonomia destes, sempre vislumbrando uma potente construção de vínculos entre trabalhadores da saúde e usuários.

Diante aos relatos do presente estudo torna-se passível de identificação que a atuação do profissional de Educação Física no Consultório na Rua reflete uma ampla e complexa área de conhecimentos, uma vez que existe a possibilidade de tratar pedagogicamente com o corpo humano em sua integralidade que vai além de uma estrutura anatomofisiopatológica.

Para Varela e Oliveira (2018), a Educação Física não possui uma estrutura sólida para atuação nos processos de cuidado em saúde mental, não há receitas, manuais, ou tarefas pré-estabelecidas (e nem deve haver). A rotina de trabalho é pautada pela necessidade do território, pelas demandas de saúde da população. Nesse sentido, torna-se oportuno frisar que é uma área de atuação em constante construção, e por isso, demanda mais estudos, mais experiências, maior investimento.

Desta forma, ressalta-se que o processo de trabalho do profissional de Educação Física deve ser denotado por ações compartilhadas, com vistas a uma intervenção interdisciplinar, que busque ampliar e fortalecer as intervenções das equipes de saúde. Além disso, o profissional deve conhecer as características do local e da população que lhe é referenciada, para atuar de acordo com as demandas de saúde (Brasil, 2008; Schuh et al., 2015)

Diante disso, torna-se fundamental que o processo de formação em Educação Física englobe a compreensão de que as dimensões socioeconômicas e culturais também são determinantes da saúde, estabelecendo diálogos na direção da saúde coletiva, visando romper com a perspectiva exclusivamente positivista da saúde, criando outras possibilidades de ação (Furtado et al., 2015).

No entanto, ainda observa-se um processo de formação em Educação Física distante das demandas dos serviços públicos de saúde. Para Anjos e Duarte (2009), as instituições de ensino superior devem dotar seus profissionais de noções de políticas públicas, gestão em saúde, instrumentos, bem como (re)configuração de suas instituições para que possam ser exercidas funções enquanto profissionais de saúde.

Não obstante, Silva e Matos (2020) apontam que nas matrizes curriculares do curso de Educação Física de instituições de ensino superior no Estado de Sergipe, são ofertadas, minimamente, disciplinas voltadas para apreensão do conhecimento frente à Saúde Pública e Coletiva, bem como estágios que não direcionam o aluno para atuação neste campo, sendo sua formação estritamente pautada em uma abordagem curativa e terapêutica, sobretudo, para grupos de riscos por meio de protocolos médicos-centrado.

Corroborando com a literatura científica, destaca-se que o fato do autor nunca ter tido contato (teórico e/ou prático) com o cenário de prática em questão, ocasionou no início da atuação notória insegurança acerca do mencionado cenário, o que dificultou um melhor envolvimento nos processos, haja vista que a formação inicial (graduação) não prepara o futuro egresso do curso de Educação Física para atuação e subsequente intervenção na saúde coletiva e, neste caso, mais precisamente, na Rede de Atenção Psicossocial.

Santos et al. (2014) apontam que apesar de a Educação Física conquistar cada vez mais seu espaço dentro da Saúde Mental e estar pautada por regulamentações, portarias e políticas públicas, geralmente o profissional desta área ainda não faz parte do corpo técnico das instituições de saúde mental.

Sinaliza-se também a falta do profissional da área (Educação Física) inserido no Consultório na Rua para acompanhar e orientar os caminhos a serem percorridos durante o processo de vivência neste serviço de saúde, por vezes, faltava à compreensão de terminologias mais específicas à saúde mental. Desta forma, entende-se que tal ausência profissional impossibilitou que o autor, no início do processo de experiência, pudesse realizar suas intervenções de forma mais elaborada e com mais dinâmica. Não obstante, Wachs, Almeida e Brandão (2016) a partir dos resultados do seu estudo evidenciaram ser frequente encontrar egressos do curso de Educação Física que trabalham no campo da saúde coletiva afirmarem que sua formação inicial (graduação) não os preparou de forma satisfatória para atuação e intervenção nesse âmbito. 


\section{Considerações Finais}

Constatou-se que a atuação do Profissional de Educação Física no Consultório na Rua submerge a reflexão sobre a formação inicial (graduação) e durante o Programa de Residência Multiprofissional em Saúde, possibilitando a aquisição de saberes teóricos e práticos frente aos processos de cuidado em saúde. Desta forma, a atuação deste profissional vai além de ações voltadas para o seu núcleo de formação, a exemplo da oferta da atividade física/práticas corporais, e desenvolve uma atuação conjunta na equipe multidisciplinar, através de intervenções voltadas para o campo de atuação, pautadas em uma perspectiva interdisciplinar.

Desta forma, a atuação no Consultório na Rua proporcionou a aquisição de conhecimentos que durante o processo de formação na graduação não foi oportunizado, dito isto, sinalizo o aprendizado frente ao apoio matricial, acolhimento, busca ativa, educação em saúde, articulação intrasetorial e intersetorial, discussões de casos, escuta ativa e estratégias de redução de danos. Haja vista que tais tecnologias e estratégias importantes para o processo de trabalho potencializam a oferta do cuidado a partir de uma perspectiva integral acerca do processo saúde-doença.

Portanto, a Residência Multiprofissional tendo como cenário de prática o Consultório na Rua, torna-se uma potente estratégia para proporcionar processos de ensino e aprendizagens com vistas a uma formação continuada, bem como apresentar o papel da Educação Física visando os processos de cuidado em saúde (levando-se em consideração os fatores determinantes e condicionantes) para a resolubilidade das problemáticas encontradas no campo da saúde coletiva/mental.

Sugerem-se mais estudos com vistas ao compartilhamento de conhecimentos acerca das possibilidades de atuação e intervenção do Profissional de Educação Física nos diversos campos da saúde coletiva tanto referente ao núcleo de especificidade como em relação à atuação em equipes multiprofissionais com enfoque nas perspectivas e desafios.

\section{Referências}

Akerman, M., \& Feuerwerker, L. (2016). Estou me formando (ou me formei) e quero trabalhar: que oportunidades o Sistema de Saú de me oferece na Saúde Coletiva? Onde posso atuar e que competências precisos desenvolver? In: Campos, G.W.S., et al. (Org.). Tratado de Saúde Coletiva: Hucitec, p. 183-198. https://professor-ruas.yolasite.com/resources/Tratado\%20de\%20Saude\%20Coletiva.pdf

Anjos, T., \& Duarte, A. C. G. O. (2009). Educação Física e a Estratégia Saúde da Família: Formação e atuação profissional. Physis, 19(4), 1127-1144. https://doi.org/10.1590/S0103-73312009000400012.

Ayres, J. R. C. M. (2006). Cuidado e humanização das práticas de saúde. In: Deslandes, S. F. (Org.). Humanização dos cuidados em saúde: conceitos, dilemas e práticas: Fiocruz, 49-84. https://www.scielo.br/scielo.php?script=sci_arttext\&pid=S0103-11042016000400312

Barros, L. M. (2015) Saúde Coletiva. Série Bibliográfica. UNIT, 1, 160.

Carvalho, Y. M. (2001). Atividade física e saúde: onde está e quem é o sujeito da relação? Revista Brasileira de Ciências do Esporte, 22(2), 9-21. http://revista.cbce.org.br/index.php/RBCE/article/view/409

Carvalho, Y. M., \& Ceccim, R. B. (2006). Formação e educação em saúde: aprendizados com a saúde coletiva. In: CAMPOS, G.W. et al (Org.). Tratado de saúde coletiva. (2a ed.): Hucitec-Fiocruz, 1, 137-70.

Constituição da República Federativa do Brasil de 1988. Brasília: Congresso Nacional. http://www.planalto.gov.br/ccivil_03/constituicao/constituicao.htm

Cruz, M. S., \& Ferreira, S. M. B. (2007). O vínculo necessário entre a saúde mental e o Programa de saúde da família na construção da rede de atenção integral aos problemas relacionados ao uso de álcool e outras drogas. In.: Cadernos IPUB, Rio de Janeiro, v.XIII, n. 24.

Decreto Presidencial n 7.053 , de 23 de dezembro de 2009. Institui a Política Nacional para a População em Situação de Rua e seu Comitê Intersetorial de Acompanhamento e Monitoramento, e dá outras providências. Diário Oficial da União, dez. Seção 1, 24. http://www.planalto.gov.br/ccivil_03/_ato20072010/2009/decreto/d7053.htm

Furtado, R. P. et al. (2015). O trabalho do professor de educação física no CAPS: aproximações iniciais. Movimento, 21(1), 4152. https://doi.org/10.22456/1982-8918.43457

Godoy, A. S. (1995). Introdução à Pesquisa Qualitativa e suas Possibilidades. Revista de Administração, 35(2), 57-63. https://www.scielo.br/pdf/rae/v35n2/a08v35n2.pdf

Goellner, S. V. (2010). A educação dos corpos, dos gêneros e das sexualidades e o reconhecimento da diversidade. Cadernos de Formação RBCE, 1(2), 71-83. http://revista.cbce.org.br/index.php/cadernos/article/view/984 
https://bvsms.saude.gov.br/bvs/publicacoes/caderno_atencao_basica_diretrizes_nasf.pdf

Junqueira, L. A. P. (2000). Intesetorialidade, transetorialidade e redes sociais na saúde. Revista de Administração Pública, 34(6), 35-45. http://bibliotecadigital.fgv.br/ojs/index.php/rap/article/view/6346.

Kessler, M. et al. (2019). Longitudinalidade do cuidado na atenção primária: avaliação na perspectiva dos usuários. Acta Paulista de Enfermagem, 32(2). 18693. http://dx.doi.org/10.1590/1982-0194201900026

Lei n. 8.080, de 19 de setembro de 1990: [Lei Orgânica da Saúde]. Diário Oficial da República Federativa do Brasil, Brasília, DF, 18.055. https://www2.camara.leg.br/legin/fed/lei/1990/lei-8080-19-setembro-1990-365093-publicacaooriginal-1-pl.html

Machado, M. et al. (2007). Integralidade, formação de saúde, educação em saúde e as propostas do SUS - Uma revisão conceitual. Revista Ciência e Saúde Coletiva, 12(2), 335-42. https://doi.org/10.1590/S1413-81232007000200009.

Melo, Z. M. (2000). Estigma: espaço para exclusão social. Revista Symposium, 4, 18-22. https://www.maxwell.vrac.puc-rio.br/2457/2457.pdf

Minayo, M. C. de S. (2004). O desafio do conhecimento: pesquisa qualitativa em saúde. (8a ed.): Hucitec.

Moura, M. S. S., \& Giannella, V. (2016). A arte de escutar: nuances de um campo de práticas e de conhecimento. Revista Terceiro Incluído, 6(1), 9-24. https://doi.org/10.5216/teri.v6i1.40739

Oliveira, B., \& Wachs, F. (2018). Educação Física, atenção primária à saúde e organização do trabalho com apoio matricial. Revista Brasileira de Ciências Esporte. Porto Alegre, 41(2). https://doi.org/10.1016/j.rbce.2018.09.003

Oliveira, C. M., Marques, V. F., \& Schreck, R. S. C. (2017). Aplicação de metodologia ativa no processo de ensino aprendizagem: relato de experiência: Estágio em Nutrição em Saúde Coletiva. Revista Eletrônica Pesquiseduca, 9(19), 674-684. https://periodicos.unisantos.br/pesquiseduca/article/view/633

Portaria GM nº154 de 24 de Janeiro de 2008. Cria os Núcleos de Apoio à Saúde da Família - NASF. Brasília: Ministério da Saúde. https://bvsms.saude.gov.br/bvs/saudelegis/gm/2008/prt0154_24_01_2008.html

Santos, F. T. et al. (2014). O papel desinstitucionalizador da educação física na saúde mental. Motrivivência, Florianópolis, 26(42), 281-292. https://doi.org/10.5007/2175-8042.2014v26n42p281

Schuh, L. et al. (2015). A inserção do profissional de educação física nas equipes multiprofissionais da estratégia saúde da família. Saúde, Santa Maria, 41(1), 29-36. http://dx.doi.org/10.5902/2236583410514

Secretaria de Atenção à Saúde. Departamento de Atenção Básica. Diretrizes do NASF: Ministério da Saúde.

Secretaria de Gestão do Trabalho e da Educação na Saúde. Departamento de Gestão da Educação na Saúde. Residência multiprofissional em saúde: experiências, avanços e desafios. Brasília. http://bvsms.saude.gov.br/bvs/publicacoes/residencia_multiprofissional.pdf

Silva, J. G., \& Matos, F. C. S. (2020). O currículo da Educação Física e a formação profissional para atuação no NASF. Revista Journal of Health Connections, 9(2). 1-14. http://revistaadmmade.estacio.br/index.php/journalhc/article/viewFile/5947/47966689

Silveira, F. (2012). A formação em Educação Física e as práticas de integralidade do cuidado na saúde coletiva. 111f. Dissertação (Mestrado em Ciências da Saúde) - Universidade Federal de Sergipe. Aracaju, 2012. https://ri.ufs.br/bitstream/riufs/3767/1/Favia_Cristina_Santos_Matos_Silveira.pdf.

Varela, S. H., \& Oliveira, B. N. (2018). Alongamento? Dinâmica? Chama o Professor de Educação Física! LICERE - Revista do Programa de PósGraduação Interdisciplinar em Estudos do Lazer, 21(1), 313-340. https://doi.org/10.35699/1981-3171.2018.1780

Vasconcelos, E. V. P. (2015). Caracterização da População em situação de rua no município de Aracaju-sergipe. Trabalho de Conclusão do Curso de Especialização em Saúde da Família Submetido à Universidade Federal de Sergipe. Universidade Aberta do SUS. https://ares.unasus.gov.br/acervo/html/ARES/12075/1/Edney\%20Vander\%20Pacheco\%20de\%20Vasconcelos\%20TCC.pdf

Vinadé, T. F., Cruz, M. S., \& Barbeito M. M. Capítulo 5. (2014). Estratégias de Redução de Danos: da atenção primária à secundária. In: Souza, M. L. O. S. Atenção Integral na rede de saúde: módulo 5. (5a ed.), Secretaria Nacional de Políticas sobre Drogas, p. 91-107.

Waal, M. et. al. (2009). Evaluation of community level interventions to address social and structural determinants of health: a cluster randomised controlled trial. BMC Public Health. 9, 9-207. https://doi:10.1186/1471-2458-9-207

Wachs, F., Almeida, U., \& Brandão, F. F. (2016). Educação física e saúde coletiva: cenários, experiências e artefatos culturais: Rede UNIDA. http://historico.redeunida.org.br/editora/biblioteca-digital/serie-interlocucoes-praticas-experiencias-e-pesquisas-em-saude/educacao-fisica-e-saude-coletivacenarios-experiencias-e-artefatos-culturais-pdf

Zappelini, L. D., \& Oliveira, C. S. (2007). O processo de acolhimento em saúde mental: construindo mudanças. Boletim da Saúde, 21(2), 67-80. https://www.scielo.br/scielo.php?pid=S1414-98932015000401340\&script=sci_abstract\&tlng=pt 